\title{
INDEX SUMMABILITY OF AN INFINITE SERIES USING $\delta$-QUASI MONOTONE SEQUENCE
}

\author{
Mahendra Misra ${ }^{1}$ B.P.Padhy ${ }^{2}$ Santosh kumar Nayak $^{3}$ \\ U.K.Misra ${ }^{4}$ \\ ${ }^{1}$ P.G.Department of Mathematics \\ N.C. College(Autonomous) \\ Jajpur, Odisha \\ ${ }^{2}$ Roland Institute of Technology \\ Golanthara-761008, Odisha, India. \\ ${ }^{3}$ Department of Mathematics \\ Jeevan Jyoti Mahavidyalaya \\ Raikia, Khandhamal, Odisha, India \\ ${ }^{4}$ Department of Mathematics \\ National Institute of Science and Technology \\ Pallur Hills, Golanthara-761008, Odisha, India
}

Keywords: Quasi-increasing, Quasi - $\mathrm{f}$ - power increasing, $\delta$ - Quasi monotone, index absolute Summability, summability factor.

ABSTRACT: A result concerning absolute indexed Summability factor of an infinite series using $\delta$ - Quasi monotone sequence has been established.

\section{INTRODUCTION:}

A positive sequence $\left(a_{n}\right)$ is said to be almost increasing if there exists a positive sequence $\left(b_{n}\right)$ and two positive constants $\mathrm{A}$ and $\mathrm{B}$ such that

$$
A b_{n} \leq a_{n} \leq B b_{n} \text {, for all } n
$$

The sequence $\left(a_{n}\right)$ is said to be quasi- $\beta$-power increasing, if there exists a constant $K$ depending upon $\beta$ with $K \geq 1$ such that

$$
K n^{\beta} a_{n} \geq m^{\beta} a_{m},
$$

for all $\mathrm{n} \geq \mathrm{m}$. In particular, if $\beta=0$, then $\left(a_{n}\right)$ is said to be quasi-increasing sequence. It is clear thatevery almost increasing sequence is a quasi- $\beta$-power increasing sequence for any non-negative $\beta$. But the converse is not true as $\left(n^{-\beta}\right)$ is quasi- $\beta$-power increasing but not almost increasing. Let $f=\left(f_{n}\right)$ be a positive sequence of numbers. Then the positive sequence $\left(a_{n}\right)$ is said to be quasi$f$-power increasing, if there exists a constant $\mathrm{K}$ depending upon $\mathrm{f}$ with $\mathrm{K} \geq 1$ such that

$$
K f_{n} a_{n} \geq f_{m} a_{m},
$$

for $\mathrm{n} \geq \mathrm{m} \geq 1([6])$. Clearly, if $\left(a_{n}\right)$ is a quasi- $f$-power increasing sequence, then the $\left(a_{n} f_{n}\right)$ is a quasiincreasing sequence. 


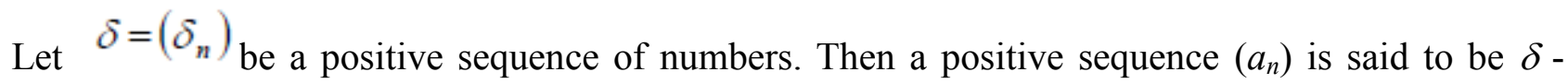
quasi monotone [1], if $a_{n} \rightarrow 0, a_{n}>0$ ultimately and $\Delta d_{n} \geq-\delta_{n}$, where $\Delta d_{n}=d_{n}-d_{n+1}$.

Let $\sum a_{n}$ be an infinite series with sequence of partial sums $\left\{\mathrm{s}_{n}\right\}$. Let $\left\{\mathrm{p}_{\mathrm{n}}\right\}$ be a sequence of positive numbers such that

$$
P_{n}=\sum_{v=0}^{n} p_{v} \rightarrow \infty, \text { as } n \rightarrow \infty
$$

Then the sequence-to-sequence transformation

$$
T_{n}=\frac{1}{P_{n}} \sum_{V=0}^{n} p_{V} s_{V}, P_{n} \neq 0,
$$

defines the $\left(\bar{N}, p_{n}\right)$ mean of the sequence $\left(s_{n}\right)$ generated by the sequence of coefficients $\left\{p_{n}\right\}$. The series $\sum a_{n}$ is said to be summable $\left|\bar{N}, p_{n}\right|_{k}, k \geq 1([2])$, if

$$
\sum_{n=1}^{\infty}\left(\frac{P_{n}}{p_{n}}\right)^{k-1}\left|T_{n}-T_{n-1}\right|^{k}<\infty
$$

The series $\sum a_{n}$ is said to be summable $\left|\bar{N}, p_{n} ; \delta\right|_{k}, k \geq 1, \delta \geq 0$, if

$$
\sum_{n=1}^{\infty}\left(\frac{P_{n}}{p_{n}}\right)^{c k+k-1}\left|T_{n}-T_{n-1}\right|^{k}<\infty .
$$

\section{KNOWN THEOREMS:}

Dealing with quasi- $\beta$-power increasing sequence Bor and Debnath [3] have established the following theorem:

\subsection{THEOREM:}

Let $\left(\mathrm{X}_{n}\right)$ be a quasi- $\beta$-power increasing sequence for $0<\beta<1$ and $\left(\lambda_{n}\right)$ be a real sequence. If the conditions

$$
\begin{aligned}
& \sum_{n=1}^{m} \frac{P_{n}}{n}=O\left(P_{m}\right), \\
& \lambda_{n} X_{n}=O(1),
\end{aligned}
$$




$$
\begin{aligned}
& \sum_{n=1}^{m} \frac{\left|t_{n}\right|^{k}}{n}=O\left(X_{m}\right), \\
& \sum_{n=1}^{m} \frac{p_{n}\left|t_{n}\right|^{k}}{P_{n}}=O\left(X_{m}\right) \\
& \sum_{n=1}^{m} n X_{n}\left|\Delta^{2} \lambda_{n}\right|<\infty
\end{aligned}
$$

are satisfied, where $t_{n}$ is the $(\mathrm{C}, 1)$ mean of the sequence $\left(n a_{n}\right)$. Then the series $\sum a_{n} \lambda_{n}$ is summable $\left|\bar{N}, p_{n}\right|_{k}, k \geq 1$

Subsequently Leindler [4] established a similar result reducing certain condition of Bor. He established:

\subsection{THEOREM:}

Let the sequence $\left(X_{n}\right)$ be a quasi- $\beta$-power increasing sequence for $0<\beta<1$, and the real sequence $\left(\lambda_{n}\right)$ satisfies the conditions

$$
\begin{aligned}
& \sum_{n=1}^{m} \lambda_{n}=O(m) \\
& \sum_{n=1}^{m}\left|\Delta \lambda_{m}\right|=O(m) .
\end{aligned}
$$

and

hold, where $X_{n}(\beta)=\max \left(n^{\beta} X_{n}, \log n\right)$. Then the series $\sum a_{n} \lambda_{n}$ is summable $\left|\bar{N}, p_{n}\right|_{k}, k \geq 1$.

Recently, extending the above results to quasi- $f$-power increasing sequence, Sulaiman [7] have established the following theorem:

\subsection{THEOREM:}

Let $f=\left(f_{n}\right)=\left(n^{\beta} \log ^{\gamma} n\right), 0 \leq \beta<1, \gamma \geq 0$ be a sequence. Let $\left(X_{n}\right)$ be a quasi- $f$-power sequence and $\left(\lambda_{n}\right)$ a sequence of constants satisfying the conditions

$$
\begin{aligned}
& \lambda_{n} \rightarrow 0 \text { ass } \rightarrow \infty, \\
& \sum_{n=1}^{\infty} n X_{n}|\Delta| \Delta \lambda_{n}||<\infty,
\end{aligned}
$$




$$
\begin{aligned}
& \left|\lambda_{n}\right| X_{n}=O(1), \\
& \sum_{n=1}^{\infty} \frac{1}{n X_{n}^{k-1}}\left|t_{n}\right|^{k}=O\left(X_{m}\right)
\end{aligned}
$$

$$
\sum_{n=1}^{\infty} \frac{p_{n}}{P_{n}} \frac{1}{X_{n}^{k-1}}\left|t_{n}\right|^{k}=O\left(X_{m}\right)
$$

where $t_{n}$ is the $(\mathrm{C}, 1)$ mean of the sequence $\left(n a_{n}\right)$. Then the series $\sum a_{n} \lambda_{n}$ is summable $\left|\bar{N}, p_{n}\right|_{k}, k \geq 1$.

Very recently, Misra et al [5] established the following theorem:

\subsection{THEOREM:}

Let $f=\left(f_{n}\right)=\left(n^{\beta}(\log n)^{\gamma}\right)$ be a sequence and $\left(X_{n}\right)$ be a quasi- $\mathrm{f}$-power sequence. Let $\left(\lambda_{n}\right)$ a sequence of constants such that

$$
\begin{aligned}
& \lambda_{n} \rightarrow 0, \text { as } n \rightarrow \infty, \\
& \sum_{n=1}^{\infty} n X_{n}|\Delta| \Delta \lambda_{n} \mid<\infty, \\
& \left|\lambda_{n}\right| X_{n}=O(1), \\
& \sum_{n=v+1}^{m}\left(\frac{P_{n}}{p_{n}}\right)^{\delta k-1} \frac{1}{P_{n-1}}=O\left(\frac{P_{m}}{p_{m}}\right)^{\delta k-1}, \\
& \sum_{n=1}^{m}\left(\frac{P_{n}}{p_{n}}\right)^{\delta k-1} \frac{\left|t_{v}\right|^{k}}{X_{v}^{k-1}}=O\left(X_{m}\right), \\
& \sum_{n=1}^{m}\left(\frac{P_{n}}{p_{n}}\right)^{\delta k} \frac{\left|t_{n}\right|^{k}}{n X_{n}^{k-1}}=O\left(X_{m}\right) .
\end{aligned}
$$

Then the series $\sum a_{n} \lambda_{n}$ is summable $\left|\bar{N}, p_{n} ; \delta\right|_{k}, k \geq 1, \delta \geq 0$.

In what follows in this paper, using $\delta$ - quasi monotone sequence, we prove the following theorem. 


\section{MAIN THEOREM}

Let $f=\left(f_{n}\right)=\left(n^{\beta}(\log n)^{\gamma}\right)$ be a sequence and $\left(X_{n}\right)$ be a quasi- $f$-power sequence. Suppose also that there exists a sequence of numbers $\left(A_{n}\right)$ such that it is $\delta$ - quasi-monotone with

$$
\begin{aligned}
& \sum n \delta_{n} X_{n}<\infty \\
& \Delta A_{n} \leq \delta_{n} \text { for all } n .
\end{aligned}
$$

Let $\left(\lambda_{n}\right)$ a sequence of constants such that

$$
\begin{aligned}
& \lambda_{n} \rightarrow 0, \text { as } n \rightarrow \infty, \\
& \left|\lambda_{n}\right| X_{n}=O(1),
\end{aligned}
$$

$$
\left|\Delta \lambda_{n}\right| \leq\left|A_{n}\right| \text { for all } n
$$

Then the series $\sum a_{n} \lambda_{n}$ is summable $\left|\bar{N}, p_{n} ; \sigma\right|_{k}, k \geq 1, \sigma \geq 0$. if

$$
\begin{aligned}
& \sum_{n=v+1}^{m}\left(\frac{P_{n}}{p_{n}}\right)^{\sigma k-1} \frac{1}{P_{n-1}}=O\left(\frac{P_{v}}{p_{v}}\right)^{\sigma k-1}, \\
& \sum_{n=1}^{m}\left(\frac{P_{n}}{p_{n}}\right)^{\sigma k-1} \frac{\left|t_{n}\right|^{k}}{X_{n}^{k-1}}=O\left(X_{m}\right), \\
& \sum_{n=1}^{m}\left(\frac{P_{n}}{p_{n}}\right)^{\sigma k} \frac{\left|t_{n}\right|^{k}}{n X_{n}^{k-1}}=O\left(X_{m}\right),
\end{aligned}
$$

where $\left(t_{n}\right)$ is the $\mathrm{n}$ th $(\mathrm{C}, 1)$ mean of the sequence $\left(n a_{n}\right)$.

In order to prove the theorem we require the following lemma.

\section{LEMMA:}

Let $f=\left(f_{n}\right)=\left(n^{\beta}(\log n)^{\gamma}\right), 0 \leq \beta<1, \gamma \geq 0$ be a sequence and $\left(X_{n}\right)$ be a quasi $-f$-power increasing sequence. Let $\left(A_{n}\right)$ be a sequence of numbers such that it is $\delta$-quasi - monotone satisfying (3.1) and (3.2). then

$$
\begin{aligned}
& n X_{n}\left|A_{n}\right|=O(1) \\
& \sum_{n=1}^{m} X_{n}\left|A_{n}\right|<\infty, \text { as } m \rightarrow \infty .
\end{aligned}
$$

and 


\subsection{PROOF OF THE LEMMA:}

As $A_{n} \rightarrow 0$ and $n^{\beta}(\log n)^{\gamma} X_{n}$ is non-decreasing, we have

$$
\begin{aligned}
n X_{n}\left|A_{n}\right|= & n^{1-\beta}(\log n)^{-\gamma}\left(n^{\beta}(\log n)^{\gamma} X_{n}\right) \sum_{v=n}^{\infty} \Delta\left|A_{v}\right| \\
& =O(1) n^{1-\beta}(\log n)^{\gamma} n \sum_{v=n}^{\infty} v^{\beta}(\log v)^{\gamma} X_{v}\left|\Delta A_{v}\right| \\
& =O(1) \sum_{v=n}^{\infty} v^{1-\beta}(\log v)^{-\gamma} v^{\beta}(\log v)^{\gamma} X_{v}\left|\Delta A_{v}\right| \\
& =O(1) \sum_{v=n}^{\infty} v X_{v}\left|\Delta A_{v}\right| . \\
& =O(1) \sum_{v=n}^{\infty} v X_{v}\left|\delta_{v}\right| \\
& =O(1)
\end{aligned}
$$

This establishes (4.1).

Next

$$
\begin{aligned}
\sum_{n=1}^{m} X_{n}\left|A_{n}\right|= & \sum_{n=1}^{m-1}\left(\sum_{r=1}^{n} X_{r}\right) \Delta\left|A_{n}\right|+\left|A_{m}\right|\left(\sum_{r=1}^{m} X_{r}\right) \\
= & O(1) \sum_{n=1}^{m-1}\left(\sum_{r=1}^{n} r^{-\beta}(\log r)^{-\gamma} r^{\beta}(\log r)^{\gamma} X_{r}\right) \Delta\left|A_{n}\right| \\
& +O(1)\left(\sum_{r=1}^{m} r^{-\beta}(\log r)^{-\gamma} r^{\beta}(\log r)^{\gamma} X_{r}\right)\left|A_{m}\right| \\
= & O(1) \sum_{n=1}^{m-1}\left(n^{\beta}(\log n)^{\gamma} X_{n}\right)\left|A_{n}\right| \sum_{r=1}^{n} r^{-\beta-\epsilon}(\log r)^{-\gamma} r^{\epsilon} \\
= & O(1) \sum_{n=1}^{m-1}\left(n^{\beta}(\log n)^{\gamma} X_{n}\right) \Delta\left|A_{n}\right| n^{\epsilon}(\log n)^{-\gamma} \sum_{r=1}^{n} r^{-\beta-\epsilon} \\
& +O(1) m^{\beta} X_{m}\left|A_{m}\right|(\log m)^{\gamma} m^{\epsilon}(\log m)^{-\gamma} \sum_{r=1}^{m} r^{-\beta-\epsilon} \\
= & O(1) \sum_{n=1}^{m} n^{\beta+\varepsilon} X_{n} \Delta\left|A_{n}\right|\left(\int_{1}^{n} u^{-\beta-\varepsilon} d u\right)+O(1) m^{\beta+\varepsilon} X_{m}\left|A_{m}\right|\left(\int_{1}^{m} u^{-\beta-\epsilon} d u\right) \\
= & O(1) \sum_{n=1}^{m} n X_{n} \Delta\left|A_{n}\right|+O(1) m X_{m}\left|A_{m}\right| \\
= & O(1) .
\end{aligned}
$$

This establishes (4.2).

\section{PROOF OF THE THEOREM:}

Let $\left(T_{n}\right)$ be the sequence of $\left(\bar{N}, p_{n}\right)$ mean of the series $\sum_{n=1}^{\infty} a_{n} \lambda_{n}$, then

$$
\begin{aligned}
& T_{n}=\frac{1}{P_{n}} \sum_{v=0}^{n} p_{v} \sum_{r=0}^{v} a_{r} \lambda_{r} \\
& =\frac{1}{P_{n}} \sum_{v=0}^{n}\left(P_{n}-P_{v-1}\right) a_{v} \lambda_{v}
\end{aligned}
$$




$$
\begin{aligned}
& \text { Hence for } \mathrm{n} \geq 1 \\
& \begin{aligned}
T_{n}-T_{n-1}= & \frac{p_{n}}{P_{n} P_{n-1}} \sum_{v=1}^{n} P_{v-1} a_{v} \lambda_{v} \\
\quad & \frac{p_{n}}{P_{n} P_{n-1}} \sum_{v=1}^{n} v a_{v}\left(\frac{1}{v} P_{v-1} \lambda_{v}\right) \\
= & \frac{(n+1)}{n} \frac{p_{n}}{P_{n}} t_{n} \lambda_{n}+\frac{p_{n}}{P_{n} P_{n-1}} \sum_{v=1}^{n-1} p_{v-1} t_{v} \lambda_{v} \frac{v+1}{v}+\frac{p_{n}}{P_{n} P_{n-1}} \sum_{v=1}^{n-1} P_{v} t_{v} \frac{v+1}{v} \Delta \lambda_{v} \\
& +\frac{p_{n}}{P_{n} P_{n-1}} \sum_{v=1}^{n-1} P_{v} t_{v} \frac{\lambda_{v+1}}{v} \\
= & T_{n 1}+T_{n 2}+T_{n 3}+T_{n 4} \text { (say). }
\end{aligned}
\end{aligned}
$$

In order to prove the theorem, using Minkowski's inequality it is enough to show that

$$
\sum_{n=1}^{\infty}\left(\frac{P_{n}}{p_{n}}\right)^{o k+k-1}\left|T_{n j}\right|<\infty \quad, j=1,2,3,4 .
$$

Applying Hölder's inequality, we have

$$
\begin{aligned}
\sum_{n=1}^{m}\left(\frac{P_{n}}{p_{n}}\right)^{o k+k-1}\left|T_{n 1}\right|^{k} & =\sum_{n=1}^{m}\left(\frac{P_{n}}{p_{n}}\right)^{o k+k-1}\left|\frac{n+1}{n} \frac{p_{n}}{P_{n}} t_{n} \lambda_{n}\right|^{k} \\
& =O(1) \sum_{n=1}^{m}\left(\frac{P_{n}}{p_{n}}\right)^{o k-1} \frac{\left|t_{n}\right|^{k}}{X_{n}^{k-1}}\left(X_{n}\left|\lambda_{n}\right|\right)^{k-1}\left|\lambda_{n}\right| \\
& =O(1) \sum_{n=1}^{m}\left(\frac{P_{n}}{p_{n}}\right)^{o k-1} \frac{\left|t_{n}\right|^{k}}{X_{n}^{k-1}}\left|\lambda_{n}\right| \\
& =O(1) \sum_{n=1}^{m-1}\left(\sum_{v=1}^{n}\left(\frac{P_{v}}{p_{v}}\right)^{o k-1} \frac{\left|t_{v}\right|^{k}}{X_{v}^{k-1}}\right) \Delta\left|\lambda_{n}\right|+O(1) \sum_{v=1}^{m}\left(\frac{P_{v}}{p_{v}}\right)^{o k-1} \frac{\left|t_{v}\right|^{k}}{X_{v}^{k-1}}\left|\lambda_{m}\right| \\
& \leq O(1) \sum_{n=1}^{m-1} X_{n}\left|A_{n}\right|+O(1) X_{m}\left|\lambda_{m}\right| \\
& =O(1) .
\end{aligned}
$$

Next

$$
\begin{aligned}
\sum_{n=1}^{m}\left(\frac{P_{n}}{p_{n}}\right)^{o k+k-1}\left|T_{n 2}\right|^{k} & =\sum_{n=1}^{m}\left(\frac{P_{n}}{p_{n}}\right)^{o k+k-1}\left|\frac{p_{n}}{P_{n} P_{n-1}} \sum_{v=1}^{n-1} p_{v-1} t_{v} \lambda_{v} \frac{v+1}{v}\right|^{k} \\
& =\left.\left.O(1) \sum_{n=1}^{m}\left(\frac{P_{n}}{p_{n}}\right)^{o k-1} \frac{1}{P_{n-1}} \sum_{v=1}^{n-1} p_{v-1}\right|_{v}\right|^{k}\left|\lambda_{v}\right|^{k}\left(\sum_{v=1}^{n-1} \frac{p_{v}}{P_{n-1}}\right)^{k-1} \\
& =O(1) \sum_{v=1}^{m} p_{v-1}\left|t_{v}\right|^{k}\left|\lambda_{v}\right|^{k} \sum_{n=v+1}^{m}\left(\frac{P_{n}}{p_{n}}\right)^{o k-1} \frac{1}{P_{n-1}} . \\
& =O(1) \sum_{v=1}^{n}\left(\frac{P_{v}}{p_{v}}\right)^{o k-1}\left|t_{v}\right|^{k}\left|\lambda_{v}\right|^{k} \\
& =O(1), \text { as in the case of } T_{n 1} .
\end{aligned}
$$

Next

$$
\sum_{n=1}^{m}\left(\frac{P_{n}}{p_{n}}\right)^{o k+k-1}\left|T_{n 3}\right|^{k}=\sum_{n=1}^{m}\left(\frac{P_{n}}{p_{n}}\right)^{o k+k-1}\left|\frac{p_{n}}{P_{n} P_{n-1}} \sum_{v=1}^{n-1} P_{v} t_{v} \frac{v+1}{v} \Delta \lambda_{v}\right|^{k}
$$




$$
\begin{aligned}
& =O(1) \sum_{n=1}^{m}\left(\frac{P_{n}}{p_{n}}\right)^{\sigma k-1} \frac{1}{P_{n-1}^{k}} \sum_{v=1}^{n-1} P_{v}^{k} \frac{\left|t_{v}\right|^{k}}{X_{v}^{k-1}}\left|\Delta \lambda_{v}\right|\left(\sum_{v=1}^{n-1} X_{v}\left|\Delta \lambda_{v}\right|\right)^{k-1} \\
& \leq O(1) \sum_{n=1}^{m}\left(\frac{P_{n}}{p_{n}}\right)^{\sigma k-1} \frac{1}{P_{n-1}^{k}} \sum_{v=1}^{n-1} P_{v}^{k} \frac{\left|t_{v}\right|^{k}}{X_{v}^{k-1}}\left|A_{v}\right|\left(\sum_{v=1}^{n-1} X_{v}\left|A_{v}\right|\right)^{k-1} \\
& =O(1) \sum_{v=1}^{m} P_{v}^{k} \frac{\left|t_{v}\right|^{k}}{X_{v}^{k-1}}\left|A_{v}\right| \sum_{n=v+1}^{m+1}\left(\frac{P_{n}}{p_{n}}\right)^{o k-1} \frac{1}{P_{n-1}^{k}} . \\
& =O(1) \sum_{v=1}^{m}\left(\frac{P_{v}}{p_{v}}\right)^{o k} \frac{1}{v} \frac{\left|t_{v}\right|^{k}}{X_{v}^{k-1}}\left(v\left|A_{v}\right|\right) \\
& =O(1) \sum_{v=1}^{m-1} \sum_{r=1}^{v}\left(\frac{P_{r}}{p_{r}}\right)^{o k} \frac{1}{r} \frac{\left|t_{r}\right|^{k}}{X_{r}^{k-1}} \Delta\left(v\left|A_{v}\right|\right)+0\left(m\left|A_{m}\right|\right) \sum_{r=1}^{m}\left(\frac{P_{r}}{p_{r}}\right)^{\sigma k} \frac{1}{r} \frac{\left|t_{r}\right|^{k}}{X_{r}^{k-1}} \\
& =O(1) \sum_{v=1}^{m-1}\left(-\left|A_{v}\right|+(v+1)|\Delta|\left|A_{v}\right|\right) X_{v}+0\left(m X_{m}\left|A_{m}\right|\right) . \\
& =O(1) \sum_{v=1}^{n} X_{v}\left|A_{v}\right|+0(1) \sum_{v=1}^{n} v X_{v}\left|\Delta A_{v}\right|+0\left(m X_{m}\left|A_{m}\right|\right) \\
& \leq O(1) \sum_{v=1}^{n} X_{v}\left|A_{v}\right|+0(1) \sum_{v=1}^{n} v X_{v} \delta_{v}+0\left(m X_{m}\left|A_{m}\right|\right) \\
& =O(1) .
\end{aligned}
$$

Finally

$$
\begin{aligned}
\sum_{n=1}^{m}\left(\frac{P_{n}}{p_{n}}\right)^{o k+k-1}\left|T_{n 4}\right|^{k} & =\sum_{n=1}^{m}\left(\frac{P_{n}}{p_{n}}\right)^{o k+k-1}\left|\frac{p_{n}}{P_{n} P_{n-1}} \sum_{v=1}^{n-1} P_{v} t_{v} \frac{\lambda_{v+1}}{v}\right|^{k} \\
& =O(1) \sum_{n=1}^{m}\left(\frac{P_{n}}{p_{n}}\right)^{o k-1} \frac{1}{P_{n-1}^{k}} \sum_{v=1}^{n-1} \frac{P_{v}}{v}\left|t_{v}\right|^{k}\left|\lambda_{v}\right|^{k}\left(\sum_{v=1}^{n-1} \frac{P_{v}}{v}\right)^{k-1} \\
& =O(1) \sum_{v=1}^{m} \frac{P_{v}}{v}\left|t_{v}\right|^{k}\left|\lambda_{v}\right|^{k} \sum_{n=v+1}^{m}\left(\frac{P_{n}}{p_{n}}\right)^{o k-1} \frac{1}{P_{n-1}} \\
& =O(1) \sum_{v=1}^{m}\left(\frac{P_{v}}{p_{v}}\right)^{o k} \frac{\left|t_{v}\right|^{k}}{X_{v}{ }^{k-1}}\left(X_{v}\left|\lambda_{v}\right|\right)^{k-1}\left|\lambda_{v}\right| \\
& =O(1) \sum_{v=1}^{m}\left(\frac{P_{v}}{p_{v}}\right)^{o k} \frac{\left|t_{v}\right|^{k}\left|\lambda_{v}\right|}{v X_{v}{ }^{k-1}} \\
& =O(1) \sum_{v=1}^{m-1}\left(\sum_{r=1}^{v}\left(\frac{P_{r}}{p_{r}}\right)^{o k} \frac{\left|t_{r}\right|^{k}}{r X_{r}{ }^{k-1}}\right)|\Delta| \lambda_{v} \mid+O(1) \sum_{r=1}^{m}\left(\frac{P_{r}}{p_{r}}\right)^{o k} \frac{\left|t_{r}\right|^{k}}{r X_{r}{ }^{k-1}} \\
\leq & O(1) \sum_{v=1}^{m-1}\left(\sum_{r=1}^{v}\left(\frac{P_{r}}{p_{r}}\right)^{o k} \frac{\left|t_{r}\right|^{k}}{r X_{r}{ }^{k-1}}\right)\left|A_{v}\right|+O(1) \sum_{r=1}^{m}\left(\frac{P_{r}}{p_{r}}\right)^{o k} \frac{\left|t_{r}\right|^{k}}{r X_{r}{ }^{k-1}} \\
& =O(1) \sum_{v=1}^{m} X_{v}\left|A_{v}\right|+O(1) X_{m}\left|\lambda_{m}\right| \\
& =O(1) .
\end{aligned}
$$

This completes the proof of the theorem. 


\section{REFERENCES}

[1]. R.P.Boas, “Quasi positive sequences and trigonometric series”, Pro. London Math. Soc.. 14A (1965), 38-46

[2]. H.Bor, “A Note on two summability methods”, Proc.Amer. Math. Soc.98 (1986), 81-84.

[3]. H.Bor and L.Debnath, "Quasi - $\beta$ - power increasing sequences", International journal of Mathematics and Mathematical Sciences,44(2004),2371-2376.

[4]. L.Leinder, "A recent note on absolute Riesz summability factors", J. Ineq. Pure and Appl. Math.,Vol-7, Issue-2,article-44(2006).

[5]. U.K.Misra, M.Misra, B.P.Padhy and D.Bisoyi, "On Quasi-f-power increasing sequences", International Mathematical Forum, Vol.8, No5-.8 (2013),377-386

[6]. W.T.Sulaiman, "Extension on absolute summability factors of infinite series", J. Math. Anal. Appl. 322 (2006), 1224-1230.

[7]. W.T.Sulaiman, "A recent note on absolute absolute Riesz summability factors of an infinite series”, J. Appl. Functional Analysis,Vol-7,no.4,381-387. 